\title{
HOMOTOPY GROUPS OF THE SPACE OF SELF-HOMOTOPY-EQUIVALENCES
}

BY

\author{
DARRYL MCCULLOUGH
}

\begin{abstract}
Let $M$ be a connected sum of $r$ closed aspherical manifolds of dimension $n>3$, and let $E M$ denote the space of self-homotopy-equivalences of $M$, with basepoint the identity map of $M$. Using obstruction theory, we calculate $\pi_{q}(E M)$ for $1<q<n-3$ and show that $\pi_{n-2}(E M)$ is not finitely-generated. As an application, for the case $n=3$ and $r>3$ we show that infinitely many generators of $\pi_{1}\left(E M^{3}, \mathrm{id}_{M}\right)$ can be realized by isotopies, to conclude that $\pi_{1}\left(\operatorname{Homeo}\left(M^{3}\right), \mathrm{id}_{M}\right)$ is not finitely-generated.
\end{abstract}

0. Introduction. Let $E X$ be the $H$-space of homotopy equivalences from $X$ to $X$, with the identity map of $X$ as basepoint. It contains the basepoint-preserving self-homotopy-equivalences $E_{0} X$, the group of homeomorphisms $\operatorname{Homeo}(X)$, and, when $X$ is a smooth manifold, the group of diffeomorphisms $\operatorname{Diff}(X)$. The inclusions of these subspaces are $H$-space homomorphisms. From knowledge of $E X$, one hopes to obtain information about these subspaces.

The groups $\pi_{0}\left(E_{0} X\right)$ and $\pi_{0}(E X)$ have been studied for various classes of spaces. It was shown by Sullivan [S] and, independently, Wilkerson [W] that when $X$ is a simply-connected finite complex, $\pi_{0}(E X)$ is finitely-presented. In contrast, Frank and Kahn [F-K] showed that for $p \geqslant 2, \pi_{0}\left(E_{0}\left(S^{1} \vee S^{p} \vee S^{2 p-1}\right)\right)$ is not finitelygenerated. There are examples of finite aspherical 4-complexes $K^{4}$ with $\pi_{0}\left(E_{0}\left(K^{4}\right)\right)$ not finitely-generated [M3].

Little is known about the homotopy groups $\pi_{i}(E X)$ for $i \geqslant 1$ except for two important cases. For $X$ an aspherical complex, Gottlieb [G] proved that $\pi_{1}(E X) \simeq$ center $\left(\pi_{1}(X)\right)$ while $\pi_{i}(E X)=0$ for $i \geqslant 2$. It follows that $\pi_{j}\left(E_{0} X\right)=0$ for $j \geqslant 1$. The other case is that of the $n$-sphere $S^{n}$, for which $\pi_{q}\left(E S^{n}\right)=\left[S^{q} ; \operatorname{Maps}\left(S^{n}, S^{n}\right)\right]$ $\cong\left[S^{q} \wedge S^{n} ; S^{n}\right] \cong \pi_{n+q}\left(S^{n}\right)$.

In this paper, I adapt the obstruction theory of Federer [F] to obtain some calculations of the homotopy groups of $E M$, where $M$ is any connected sum of $r>2$ (closed) aspherical (combinatorial) manifolds of dimension $n>3$. Specifically:

(1) For $1 \leqslant q \leqslant n-4, \pi_{q} E M \cong \bigoplus_{i=1}^{r-1} \pi_{n+q}\left(S^{n-1}\right)$, hence is finite.

Received by the editors April 17, 1979 and, in revised form, January 3, 1980.

The results in this paper were presented January 7, 1979 at the Geometric Topology Conference at the University of California at Berkeley.

AMS (MOS) subject classifications (1970). Primary 55D10, 57A65, 57F99, 58D05; Secondary 55B25, 55G35, 55G37.

Key words and phrases. Self-homotopy-equivalence, homeomorphism group, obstruction theory, aspherical manifold, 3-manifold, isotopy. 
(2) For $n \geqslant 4, \pi_{n-3} E M$ is a quotient of $\bigoplus_{i=1}^{r-1} \pi_{2 n-3}\left(S^{n-1}\right)$, and is finite.

(3) $\pi_{n-2} E M$ is infinitely-generated as an abelian group.

For the case $n=3$ and $r \geqslant 3$, I show that infinitely many of the generators of $\pi_{1}(E M)$ can be realized as isotopies (which can be taken to be diffeotopies) of $M$. Therefore:

(4) For $n=3$ and $r \geqslant 3, \pi_{1}\left(\operatorname{Homeo}(M), \operatorname{id}_{M}\right)$ and $\pi_{1}\left(\operatorname{Diff}(M), \mathrm{id}_{M}\right)$ are infinitely-generated.

The construction of these isotopies is very explicit. The results (1), (2), and (3) appeared in my dissertation, submitted in partial fulfillment of the requirements for the degree of Doctor of Philosophy at the University of Michigan. I wish to thank my advisor Professor Frank Raymond for his patient encouragement and helpful suggestions. I also wish to thank the referee for suggesting several significant improvements to the manuscript of this paper.

Here is a description of the program I will use to make these calculations. Let $Y$ be a CW complex and let $Y^{k}$ denote its $k$-skeleton. If $A$ and $B$ are subcomplexes with $B \subset A \subset Y$, let $(Y ; A, B)$ be the space of continuous maps from $A$ to $Y$ which restrict to the inclusion map on $B$. The inclusion map of $A$ is the basepoint of $(Y ; A, B)$. Let $Y^{[A, B]} \subset(Y ; A, B)$ be the subspace of maps which extend to all of $Y$. Because $A$ has the Homotopy Extension Property in $Y, Y^{[A, B]}$ consists of path components of $(Y ; A, B)$. There are three fibrations in which the projection maps are restriction:

$$
\begin{gathered}
Y^{\left[Y, Y^{2}\right]} \rightarrow Y^{[Y, \varnothing]} \rightarrow Y^{\left[Y^{2}, \varnothing\right]}, Y^{\left[Y, Y^{2}\right]} \rightarrow Y^{\left[Y, Y^{1}\right]} \rightarrow Y^{\left[Y^{2}, Y^{1}\right]}, \\
Y^{\left[Y^{2}, Y^{1}\right]} \rightarrow Y^{\left[Y^{2}, \varnothing\right]} \rightarrow Y^{\left[Y^{1}, \varnothing\right]} .
\end{gathered}
$$

These fit into the following diagram in which the row and columns are fibrations:

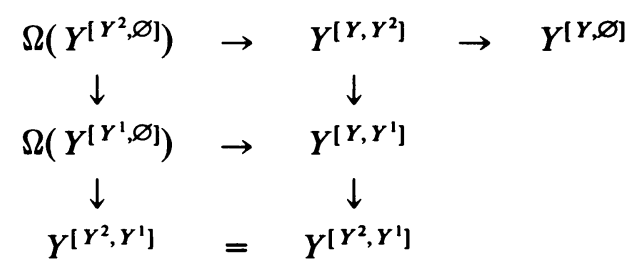

It is easy to produce from this diagram a long exact sequence:

$$
\ldots \rightarrow J_{q} \stackrel{D_{q}}{\rightarrow} K_{q} \rightarrow \pi_{q}\left(Y^{[Y, \varnothing]}\right) \rightarrow J_{q-1} \stackrel{D_{q-1}}{\rightarrow} K_{q-1} \rightarrow \ldots
$$

where

$$
\begin{aligned}
& J_{q}=\operatorname{coker}\left(\pi_{q+1}\left(Y^{\left[Y^{2}, Y^{1}\right]}\right) \rightarrow \pi_{q+1}\left(Y^{\left[Y^{2}, \varnothing\right]}\right)\right), \\
& K_{q}=\operatorname{coker}\left(\partial: \pi_{q+1}\left(Y^{\left[Y^{2}, Y^{1}\right]}\right) \rightarrow \pi_{q}\left(Y^{\left[Y, Y^{2}\right]}\right)\right),
\end{aligned}
$$

and $D_{q}$ is induced by $\partial: \pi_{q+1}\left(Y^{\left[Y^{2}, \varnothing\right]}\right) \rightarrow \pi_{q}\left(Y^{\left[Y, Y^{2}\right]}\right)$.

In $\S 1$, under certain assumptions on $Y$, we will identify $J_{q}$ and $K_{q}$ as cohomology modules of $Y$ and discuss the boundary homomorphism $D_{q}$. In $\$ 2$, we list the properties of connected sums of aspherical manifolds which allow explicit calculations of the modules to be made. The results called (1), (2) and (3) above are obtained in $\S 3$, and the isotopies of 3-manifolds are constructed in the final section. 
1. Obstruction theory preliminaries. We will denote by $I^{q}$ the $q$-dimensional cube $[0,1]^{q}$, by $J^{q}$ the closure of the complement of $I^{q} \times\{0\}$ in $\partial I^{q+1}$.

1.A. The boundary homomorphism for fibrations of spaces of mappings. Suppose that $C \subset B \subset A$ are subcomplexes of the $C W$ complex $Y$. For the fibration $Y^{[A, B]} \rightarrow Y^{[A, C]} \rightarrow Y^{[B, C]}$ the boundary homomorphism $\partial: \pi_{q+1}\left(Y^{[B, C]}\right) \rightarrow \pi_{q}\left(Y^{[A, B]}\right)$ can be described as follows [F, pp. 346-347]. Let $\langle w\rangle \in \pi_{q+1}\left(Y^{[B, C]}\right)$; then $w$ is defined on the subset $B \times I^{q} \times I \subset Y \times I^{q+1}$. Extend $w$ to $A \times J^{q} \cup B \times I^{q+1}$ using the projection map to $A$. By the Homotopy Extension Property applied to the pair $\left(A \times I^{q} \times\{1\}, A \times \partial I^{q} \times\{1\} \cup B \times I^{q} \times\{1\}\right)$, we obtain an extension to all of $A \times I^{q+1}$. If $u$ denotes the restriction of this extension to $A \times I^{q} \times\{0\}$, then $\langle u\rangle=\partial\langle w\rangle$.

1.B. Calculation of $J_{q}=\operatorname{coker}\left(\pi_{q+1}\left(Y^{\left[Y^{2}, Y^{1}\right]}\right) \rightarrow \pi_{q+1}\left(Y^{\left[Y^{2}, \varnothing\right]}\right)\right)$. All cochains and cohomology will be with local coefficients. We will denote by proj $_{y}$ the projection map from $Y \times I^{q+1}$ to $Y$, or its restriction to any subspace of $Y \times I^{q+1}$. Let * $\in Y^{0}$ be the basepoint of $Y$.

LEMMA 1.B.1. If $\pi_{q+1}\left(Y^{\left[Y^{2}, *\right]}\right) \rightarrow \pi_{q+1}\left(Y^{\left[Y^{2}, \varnothing\right]}\right)$ is surjective, then

$$
J_{q} \cong H^{1}\left(Y ; \pi_{q+2} Y\right) \text {. }
$$

Proof. Let $\langle f\rangle \in \pi_{q+1}\left(Y^{\left[Y^{2}, \varnothing\right]}\right)$; then $\left.f\right|_{Y^{2} \times \partial I^{q+1}}=\operatorname{proj}_{y}$. By assumption we may choose $f$ so that $\left.f\right|_{Y^{0} \times I^{q+1}}=\operatorname{proj}_{y}$. Consider the difference cochain $d_{q+2}\left(\operatorname{proj}_{y}, f\right) \in$ $C^{q+2}\left(Y^{2} \times I^{q+1}, Y^{2} \times \partial I^{q+1} ; \pi_{q+2} Y\right) \cong C^{1}\left(Y ; \pi_{q+2} Y\right)$. We have $\delta d_{q+2}\left(\operatorname{proj}_{y}, f\right)$ $=c_{q+3}\left(\operatorname{proj}_{y}\right)-c_{q+3}(f)=0$ since both $\operatorname{proj}_{y}$ and $f$ admit extensions to $Y^{2} \times$ $I^{q+2}$. Thus we may define $d_{1}\langle f\rangle=\left\{d_{q+2}\left(\operatorname{proj}_{y}, f\right)\right\} \in H^{1}\left(Y ; \pi_{q+2} Y\right)$. Changing $f$ by a homotopy on $Y^{0} \times I^{q+1}$ alters $d_{q+2}\left(\operatorname{proj}_{y}, f\right)$ by a coboundary so $d_{1}$ is well defined, and it is easy to see that $d_{1}$ is a homomorphism which vanishes on image $\left(\pi_{q+1}\left(Y^{\left[Y^{2}, Y^{1}\right]}\right)\right)$. If $d_{1}\langle f\rangle=0$, then $\left.f\right|_{Y^{1} \times I^{q+1}}$ is homotopic to $\operatorname{proj}_{y}$, so $\langle f\rangle \in$ image $\left(\pi_{q+1}\left(Y^{\left[Y^{2}, Y^{1}\right]}\right)\right)$; thus $d_{1}: J_{q} \rightarrow H^{1}\left(Y ; \pi_{q+2} Y\right)$ is injective. Given $\{c\} \in$ $H^{1}\left(Y ; \pi_{q+2} Y\right)$, define $f: Y^{1} \times I^{q+1} \rightarrow Y$ so that $\left.f\right|_{Y^{0} \times I^{q+1}}=\operatorname{proj}_{y}$ and $\left\langle\left. f\right|_{\sigma \times I^{q+1}}\right\rangle$ $=c(\sigma)$ for each $\sigma \in Y^{1}-Y^{0}$. Since $\delta c=0, f$ extends to $Y^{2} \times I^{q+1}$, and $d_{1}\langle f\rangle=$ $\{c\}$. Therefore $d_{1}$ is surjective.

1.C. Calculation of $K_{q}=\operatorname{coker}\left(\partial: \pi_{q+1}\left(Y^{\left[Y^{2}, Y^{1}\right]}\right) \rightarrow \pi_{q}\left(Y^{\left[Y, Y^{2}\right]}\right)\right)$.

LEMMA 1.C.1. If $H^{p}\left(Y ; \pi_{p+q} Y\right)=H^{p-1}\left(Y ; \pi_{p+q} Y\right)=0$ for $3<p<n-1$, then $K_{q} \cong H^{n}\left(Y ; \pi_{n+q} Y\right)$.

Proof. Define $d_{n}: K_{q} \rightarrow H^{n}\left(Y ; \pi_{n+q} Y\right)$ as follows. Let $\langle f\rangle$ represent an element of $K_{q}$. If $n=3$, let $d_{n}\langle f\rangle=\left\{d_{n+q}\left(\operatorname{proj}_{y}, f\right)\right\}$. Suppose $n>3$. Then $\delta d_{q+3}\left(\operatorname{proj}_{y}, f\right)$ $=c_{q+4}\left(\operatorname{proj}_{y}\right)-c_{q+4}(f)=0$ and $\left\{d_{q+3}\left(\operatorname{proj}_{y}, f\right)\right\} \in H^{3}\left(Y ; \pi_{q+3} Y\right)=0$. Hence there is a homotopy $F: f \simeq f_{1}\left(\operatorname{rel} Y^{1} \times I^{q}\right)$ with $\left.f_{1}\right|_{Y^{3} \times I^{q}}=\operatorname{proj}_{y}$. Let $g: Y^{2} \times$ $I^{q+1} \rightarrow Y$ be $\left.F\right|_{Y^{2} \times\left(I^{q} \times I\right)}$. Then $g$ represents an element of $\pi_{q+1}\left(Y^{\left[Y^{2}, Y^{1}\right]}\right)$ such that $d_{q+3}\left(\operatorname{proj}_{y}, \partial\langle g\rangle\right)=d_{q+3}\left(\operatorname{proj}_{y}, f\right)$. Moreover $\left\langle f_{1}\right\rangle=\langle f\rangle-\partial\langle g\rangle$ so $\left\langle f_{1}\right\rangle$ represents the same element of $K_{q}$ as $\langle f\rangle$ did. Inductively, for $4<k<n-1$, assume $\left.f_{1}\right|_{Y^{k-1} \times 1^{q}}=\operatorname{proj}_{y}$. We have $\left\{d_{k+q}\left(\operatorname{proj}_{y}, f_{1}\right)\right\} \in H^{k}\left(Y, \pi_{k+q} Y\right)=0$. Therefore $f_{1}$ is homotopic to a map, again called $f_{1}$, such that $\left.f_{1}\right|_{Y^{k} \times I^{q}}=$ proj $_{y}$. This completes the induction. Let $d_{n}\langle f\rangle=\left\{d_{n+q}\left(\operatorname{proj}_{y}, f_{1}\right)\right\} \in H^{n}\left(Y ; \pi_{n+q} Y\right)$. 
We must show this assignment is well defined. Suppose $\left\langle f_{1}^{\prime}\right\rangle$ is another homotopy class with $\left.f_{1}^{\prime}\right|_{Y^{n-1} \times I^{q}}=\operatorname{proj}_{y}$ and $f_{1}^{\prime} \simeq f\left(\right.$ rel $\left.Y^{1} \times I^{q}\right)$. Then $f_{1} \simeq f \simeq f_{1}^{\prime}$ (rel $\left.Y^{1} \times I^{q}\right)$ and we must show $f_{1} \simeq f_{1}^{\prime}\left(\operatorname{rel} Y^{n-2} \times I^{q}\right)$. For $n=3$, this is automatic, so assume $n>3$. Let $G: Y \times I^{q} \times I \rightarrow Y$ be a homotopy from $f_{1}$ to $f_{1}^{\prime}$. Inductively, for $2 \leqslant k \leqslant n-2$, suppose $\left.G\right|_{Y^{k-1} \times I^{q+1}}=\operatorname{proj}_{y}$. Then $\left\{d_{(q+1)+k}\left(\operatorname{proj}_{y}, G\right)\right\} \in H^{k}\left(Y ; \pi_{(q+1)+k} Y\right)=0$ so $G$ is homotopic (rel $\left.Y \times \partial I^{q+1}\right)$ to a new homotopy, also called $G$, with $\left.G\right|_{Y^{k} \times I^{q+1}}=$ proj $_{y}$. This completes the induction; thus $f_{1} \simeq f_{1}^{\prime}\left(\right.$ rel $\left.Y^{n-2} \times I^{q}\right)$ so $d_{n}\left\langle f_{1}\right\rangle=d_{n}\left\langle f_{1}^{\prime}\right\rangle$.

Clearly, $d_{n}$ is a surjective homomorphism. It remains to show $d_{n}$ is injective. Suppose $d_{n}\langle f\rangle=0$. By the preceding argument, we can find $\left\langle f_{1}\right\rangle$ with $\left.f_{1}\right|_{Y^{n-1} \times I^{q}}$ $=\operatorname{proj}_{y}, d_{n}\left\langle f_{1}\right\rangle=0$, and $f \simeq f_{1}\left(\right.$ rel $\left.Y^{1} \times I^{q}\right)$. Let $H: f \simeq f_{1} \simeq \operatorname{proj}_{y}\left(\right.$ rel $\left.Y^{1} \times I^{q}\right)$. Letting $g=\left.H\right|_{Y^{2} \times I^{q+1}}$, we have $\langle f\rangle=\partial\langle g\rangle$, so $\langle f\rangle$ represents the zero element of $K_{q}$.

1.D. An important example. The following example illustrates the techniques we use for computing homotopy groups of mapping spaces, and it is pertinent to the manifolds we will be considering. Let $X=S^{n-1} \times I$. We regard it as a cell complex with six cells: two 0 -cells $* \times\{0\}$ and $* \times\{1\}$, one 1-cell $\sigma=* \times I$ connecting the 0 -cells, two $(n-1)$-cells $S^{n-1} \times\{0\}$ and $S^{n-1} \times\{1\}$, and one $n$-cell $\tau$. Letting $C=\partial X, B=\sigma \cup \partial X$, and $A=X$, the fibration of $\S 1$.A becomes

$$
X^{[X, \sigma \cup \partial X]} \rightarrow X^{[X, \partial X]} \rightarrow X^{[\sigma \cup \partial X, \partial X]} .
$$

It is not difficult to observe that $X^{[\sigma \cup \partial X, \partial X]}=X^{[\sigma, \partial \sigma]} \simeq \Omega X$ and, because the attaching map of $\tau$ is null-homotopic, that $X^{[X, \sigma \cup \partial X]} \simeq \Omega^{n} X$. Therefore the homotopy exact sequence for the fibration becomes

$$
\ldots \rightarrow \pi_{q+2}(X) \stackrel{D_{q}}{\rightarrow} \pi_{n+q}(X) \rightarrow \pi_{q}\left(X^{[X, \partial X]}\right) \rightarrow \pi_{q+1}(X) \stackrel{D_{q-1}}{\rightarrow} \pi_{n+q-1}(X) \rightarrow \ldots
$$

It is a lengthy exercise (written out in [M2]) to check that, up to sign, $D_{q}(u)$ equals the Whitehead product $[z, u]$ where $z$ is a generator of $\pi_{n-1}(X) \simeq \mathbf{Z}$.

For the calculations of $\S \S 3$ and 4 , we should describe the isomorphisms $d_{1}$ : $\pi_{q+1}\left(X^{[\sigma \cup \partial X, \partial X]}\right) \cong \pi_{q+2}(X)$ and $d_{n}: \pi_{q}\left(X^{[X, \sigma \cup \partial X]}\right) \cong \pi_{n+q}(X)$ more explicitly. Let $f$ : $(\sigma \cup \partial X) \times I^{q+1} \rightarrow X$ represent an element of $\pi_{q+1}\left(X^{[\sigma \cup \partial X, \partial X)}\right)$; then the restriction of $f$ to $\partial X \times I^{q+1} \cup(\sigma \cup \partial X) \times \partial I^{q+1}$ equals the projection map to $X$. We define $d_{1}\langle f\rangle$ to be the value of the difference cochain $d_{q+2}\left(f, \operatorname{proj}_{X}\right)$ on the $(q+2)$-cell $\sigma \times I^{q+1}$. The definition of $d_{n}$ is similar.

2. Connected sums of aspherical manifolds. The letter $M$ will always denote a connected sum $M_{1} \# M_{2} \# \cdots \# M_{r}$ of $r \geqslant 2$ closed aspherical manifolds of dimension $n \geqslant 3$. We note that $\pi_{1} M=\pi_{1} M_{1} * \pi_{1} M_{2} * \cdots * \pi_{1} M_{r}$ is torsionfree, since each $\pi_{1} M_{i}$ is (being the fundamental group of a finite-dimensional aspherical complex).

2.A. The homotopy groups of $M$. We will state some results and notation to be used later. Except where otherwise noted, detailed proofs may be found in [M1].

The following theorem extends Bloomberg's [B] description of the universal cover of a connected sum. 
THEOREM 2.A.1 The universal cover $\tilde{M}$ of $M$ is homotopy-equivalent to a 1-point union $\bigvee_{i=1}^{r-1} \bigvee_{g \in \pi_{1} M} S_{g}^{i}$ of $(n-1)$-spheres. Furthermore, the action of $\pi_{1} M$ on $\tilde{M}$ corresponds to the left permutation action of $\pi_{1} M$ on the indices. That is, $g_{1} \in \pi_{1} M$ sends $S_{g}^{i}$ homeomorphically to $S_{g_{1}}^{i}$.

We will use $e$ to denote the identity element of a group $\pi$.

Definition. A Z-module $A=A_{e}$ is called a $\pi$-basis for a $\mathbf{Z} \pi$-module $N$ if

1. $N \cong \bigoplus_{g \in \pi} A_{g}$

2. $g: A_{e} \stackrel{\approx}{\rightarrow} A_{g}$ is the action of $\pi$ on $A_{e} \subset N$.

It follows that $g: A_{h} \stackrel{\approx}{\rightarrow} A_{g h}$ for all $g, h \in \pi$, and that any element of $N$ can be written uniquely (up to order of summands) as $\sum_{i=1}^{s} g_{i} a_{i}$, where $g_{i} \in \pi, a_{i} \in A_{e}$. Let $X$ be a connected simplicial complex with universal cover $\tilde{X}$. Let $\pi=\pi_{1} X$ and denote by $H_{f}^{i}(X ; N)$ the $i$ th cohomology of $X$ with local coefficients in $N$ (and finite cochains). The following lemma is standard for the case $N=\mathbf{Z} \pi, A_{e}=\mathbf{Z} \cdot e$.

LEMMA 2.A.2. (a) $H_{f}^{i}(X ; N) \cong H_{f}^{i}(\tilde{X} ; A)$,

(b) $H_{i}(X ; N) \cong H_{i}(\tilde{X} ; A)$.

The proof parallels the proof of the standard case (for details, see the appendix of [M2]). Using Theorem 2.A.1 and Lemma 2.A.2(a) together with Poincaré Duality in $\tilde{M}$, one obtains

Lemma 2.A.3. Let $q$ be a dimension in which $\pi_{q} M$ has a $\pi_{1} M$-basis $A_{q}$. Then

(a) $H^{1}\left(M ; \pi_{q} M\right) \cong \bigoplus_{i=1}^{r-1} \pi_{q} M$.

(b) $H^{j}\left(M ; \pi_{q} M\right)=0$ for $2 \leqslant j \leqslant n-1$.

(c) $H^{n}\left(M ; \pi_{q} M\right) \cong A_{q}$.

We will first describe $A_{q}$ for $2 \leqslant q \leqslant 2 n-4$. Order the elements of $\pi$ arbitrarily as $g_{1}, g_{2}, \ldots$ For $k \geqslant 1$ let $T_{k}=\bigvee_{j=1}^{k} \bigvee_{i=1}^{r-1} S_{g_{j}}^{i} \subset \bigvee_{g \in \pi} \bigvee_{i=1}^{r-1} S_{g}^{i}=T$. Then for all $m \geqslant 2, \pi_{m}(M, *) \cong \pi_{m}(T) \cong$ ind $\lim _{k} \pi_{m}\left(T_{k}\right)$. According to Hilton [H2], $\pi_{q}\left(T_{k}\right) \cong \bigoplus_{j=1}^{k} \bigoplus_{i=1}^{r-1} \pi_{q}\left(S_{g}^{i}\right)$ and thus $\pi_{q}(T) \cong \bigoplus_{g \in \pi} \bigoplus_{i=1}^{r-1} \pi_{q}\left(S_{g}^{i}\right)$. Since $g_{1} S_{g}^{i}=$ $S_{g_{1} g}^{i}$, it is clear that $A_{q}=\bigoplus_{i=1}^{r-1} \pi_{q}\left(S_{e}^{i}\right)$ is a $\pi$-basis for $\pi_{q} T \cong \pi_{q}(M)$.

In dimensions $2 n-3 \leqslant q \leqslant 3 n-6$ the first Whitehead products appear. As above, we have $\pi_{n-1} M \cong \bigoplus_{i=1}^{r-1} \bigoplus_{g \in \pi} \pi_{n-1}\left(S_{g}^{i}\right)$, and we may choose generators $z_{g}^{i}$ of $\pi_{n-1}\left(S_{g}^{i}\right)$ so that $g_{1} z_{g}^{i}=z_{g_{1} g}^{i}$. For each $(\alpha, \beta) \in \pi \times \pi$ and $1<i, j<r-1$, let $z_{\alpha, \beta}^{i, j}$ be a generator of $\pi_{2 n-3}\left(S_{\alpha, \beta}^{i, j}\right)$, where $S_{\alpha, \beta}^{i, j}$ is a copy of the $(2 n-3)$-sphere mapped to $T$ in such a way that the induced homomorphism sends $z_{\alpha, \beta}^{i, j}$ to the Whitehead product $\left[z_{\alpha}^{i}, z_{\beta}^{j}\right]$. We will always exclude the case of both $i=j$ and $\alpha=\beta$. In all the remaining cases, according to Hilton [H2], the image of $\pi_{m}\left(S_{\alpha, \beta}^{i, j}\right)$ is a direct summand of $\pi_{m} T$ for all $m$, and it will be regarded as a subgroup. Moreover, using direct limits again, there is a direct sum decomposition when $2 n-3 \leqslant q \leqslant 3 n-6$ :

$$
\begin{aligned}
& \pi_{q}(T) \cong \bigoplus_{g \in \pi} \bigoplus_{i=1}^{r-1} \pi_{q}\left(S_{g}^{i}\right) \oplus \underset{1<i<j}{\bigoplus} \bigoplus_{l=1}^{r-1} \pi_{q}\left(S_{g_{i}, g_{j}}^{l, l}\right) \\
& \bigoplus \underset{(\alpha, \beta) \in \pi \times \pi}{\bigoplus} \underset{1<k<l<r-1}{\bigoplus} \pi_{q}\left(S_{\alpha, \beta}^{k, l}\right) .
\end{aligned}
$$


Since $\left[z_{\alpha}^{i}, z_{\beta}^{j}\right]=(-1)^{n-1}\left[z_{\beta}^{j}, z_{\alpha}^{i}\right]$ there are commutative diagrams for all $m$ :

$$
\begin{aligned}
& \pi_{m}\left(S_{\alpha, \beta}^{i, j}\right) \rightarrow \pi_{m}(\tilde{M}) \\
& (-1)^{n-1} \downarrow \quad \nearrow \\
& \pi_{m}\left(S_{\beta, \alpha}^{j, i}\right)
\end{aligned}
$$

The action of $\pi$ on $\pi_{2 n-3}(\tilde{M})$ satisfies $g \cdot z_{\alpha, \beta}^{l, m}=z_{g \alpha, g \beta}^{l, m}$. Now let $\Gamma$ be a subset of $\pi$ having the following properties:

1. $e \notin \Gamma$.

2. For every $g \in \pi$ with $g \neq e$, exactly one of $g$ and $g^{-1}$ is contained in $\Gamma$.

Since $\pi$ is torsion-free, the second condition makes sense. In [M1] the following was proved.

\begin{tabular}{|c|c|c|}
\hline range of $q$ & $r=2$ & $r>2$ \\
\hline $2<q \leqslant n-2$ & 0 & 0 \\
\hline$n-1 \leqslant q \leqslant 2 n-4$ & $\pi_{q}\left(S_{e}\right)$ & $\bigoplus_{i=1}^{r-1} \pi_{q}\left(S_{e}^{i}\right)$ \\
\hline $2 n-3 \leqslant q \leqslant 3 n-6$ & $\pi_{q}\left(S_{e}\right) \oplus \underset{g \in \Gamma}{\bigoplus} \pi_{q}\left(S_{e, g}\right)$ & $\begin{array}{c}\bigoplus_{i=1}^{r-1} \pi_{q}\left(S_{e}^{i}\right) \oplus \underset{g \in \Gamma}{\oplus} \bigoplus_{l=1}^{r-1} \pi_{q}\left(S_{e, g}^{l, l}\right) \\
\bigoplus \bigoplus_{g \in \pi_{1} M} \bigoplus_{1<i<j<r-1}^{\bigoplus} \pi_{q}\left(S_{e, g}^{i, j}\right)\end{array}$ \\
\hline
\end{tabular}

LEMMA 2.A.4. For $2 \leqslant q \leqslant 3 n-6, \pi_{q} M$ has $a$-basis $A_{q}$ given in the following table:

We will also need the following observation, immediate from Theorem 2.A.1 and the fact that $\pi_{1} M$ is infinite.

LemMa 2.A.5. Let $q \geqslant 2$. For every nonzero $x$ in $\pi_{q} M$, there is a $g$ in $\pi_{1} M$ such that $g x \neq x$.

2.B. The relation between $E_{0} M$ and $E M . M^{k}$ will denote the $k$-skeleton of $M$. The evaluation map ev: $f \rightarrow f(*)$ gives a surjection from $E M$ to $M$ which is a fibration with fiber $E_{0} M$.

THEOREM 2.B.1. The exact homotopy sequence for the fibration $E_{0} M \rightarrow E M \rightarrow M$ decomposes into short exact sequences for every $q>1$ :

$$
0 \rightarrow \pi_{q+1} M \rightarrow \pi_{q} E_{0} M \rightarrow \pi_{q} E M \rightarrow 0 .
$$

REMARK. This holds for $q=0$ also, since $\pi_{1} M$ is centerless.

ProOF OF THE THEOREM. This will be a consequence of

Lemma 2.B.2. Suppose $g$ : $M^{1} \times I^{q} \rightarrow M$ and $\left.g\right|_{M^{1} \times \partial I^{q}}=\operatorname{proj}_{M}$. Then $g$ is homotopic (rel $\left.M^{1} \times \partial I^{q}\right)$ to a map $g_{1}$ with $\left.g_{1}\right|_{M^{0} \times I^{q}}=\operatorname{proj}_{M}$.

Deferring the proof of the lemma for a moment, we consider an element $\langle f\rangle \in \pi_{q} E M$. Then $f: M \times I^{q} \rightarrow M$ with $\left.f\right|_{M \times \partial I^{q}}=\operatorname{proj}_{M}$. Applying the lemma 
to $\left.f\right|_{M^{1} \times I^{q}}$, we can homotop $\left.f\right|_{M^{1} \times I^{q}}$ and hence $f\left(\right.$ rel $\left.M \times \partial I^{q}\right)$ so that $\left.f\right|_{* \times I^{q}}=$ $\operatorname{proj}_{M}$. Thus ev $\langle f\rangle=\left\langle f\left(* \times I^{q}\right)\right\rangle=0 \in \pi_{q} M$.

Proof of Lemma 2.B.2. For $q=1,\langle f(* \times I)\rangle$ is central in $\pi_{1}(M, *)$, which has trivial center, and the result follows easily. Assume $q>2$. Consider $d_{q}=$ $d_{q}\left(\operatorname{proj}_{M}, g\right) \in C^{q}\left(M \times I^{q} ; \pi_{q}(M)\right)$. We have $\delta d_{q}=c_{q+1}\left(\operatorname{proj}_{M}\right)-c_{q+1}(g)=0$ since both extend to the $(q+1)$-skeleton. We will show that $\delta d_{q}=0$ only if $d_{q}\left(\left[* \times I^{q}\right]\right)=0$.

We may assume that the paths used to define the local coefficient system are the unique paths in some maximal tree in the 1-skeleton of $M$. Let $\sigma$ be a 1-simplex in the tree with $\partial \sigma=\tau-*$. Then $0=\delta d_{q}\left[\sigma \times I^{q}\right]=d_{q}\left[\tau \times I^{q}\right]-d_{q}\left[* \times I^{q}\right]$; hence $d_{q}\left[\tau \times I^{q}\right]=d_{q}\left[* \times I^{q}\right]$. By induction on the distance of $\tau$ from $*$ in the maximal tree, we have $d_{q}\left[\tau \times I^{q}\right]=d_{q}\left[* \times I^{q}\right]$ for every 0 -simplex $\tau$ of $M$.

Now suppose $\sigma$ is any 1-simplex not in the maximal tree, representing an element $g_{\sigma} \in \pi_{1} M$. Then $0=\delta d_{q}\left[\sigma \times I^{q}\right]=g_{\sigma} d_{q}\left[\sigma(1) \times I^{q}\right]-d_{q}\left[\sigma(0) \times I^{q}\right]$ so $d_{q}\left[* \times I^{q}\right]$ $=g_{\sigma} d_{q}\left[* \times I^{q}\right]$. Therefore $g d_{q}\left[* \times I^{q}\right]=d_{q}\left[* \times I^{q}\right]$ for every $g \in \pi_{1} M$. By Lemma 2.A.5, this implies $d_{q}\left[* \times I^{q}\right]=d_{q}\left[\tau \times I^{q}\right]=0$ for every $\tau \in M^{0}$. Therefore the image of $\tau \times I^{q}$ is a null-homotopic $q$-sphere based at $\tau$, so we can homotop $f\left(\operatorname{rel} M \times \partial I^{q}\right)$ so that $\left.f\right|_{M^{0} \times I^{q}}=\operatorname{proj}_{M}$, which was to be proved.

2.C. $A$ cell structure for $M$. We describe a cell structure for $M$ that will facilitate our calculations. For $1 \leqslant i \leqslant r$ let $M_{i}^{\prime}=M_{i}$-open ball, and let $S_{i}=\partial M_{i}^{\prime}$. For $1 \leqslant i \leqslant r-1$ let $X_{i}=S^{n-1} \times I$ be a collar neighborhood of $S_{i}$ in $M_{i}^{\prime}$, so that $S_{i}=S^{n-1} \times\{0\}$. Give each $X_{i}$ a cell structure as in $\S 1$.D. Let $\sigma_{i}, 1<i<r-1$, be the 1-cell in $X_{i}$, and assume that $\sigma_{i} \cap S_{i}$ is the basepoint of $M_{i}^{\prime}$. Give the rest of $M_{i}^{\prime}$ any triangulation, for $1 \leqslant i \leqslant r-1$, and give $M_{r}^{\prime}$ any triangulation. Form the 1-point union of the $M_{i}^{\prime}$ for $1 \leqslant i \leqslant r-1$, and glue $S_{r}$ to its boundary $\bigvee_{i=1}^{r-1} S_{i}$ to form $M$.

The convenience of this construction stems from the following observation. From the proof of Theorem 2.A.1, the inclusion $\bigvee_{i=1}^{r-1} S_{i} \rightarrow M$ sends $\pi_{n-1}\left(\bigvee_{i=1}^{r-1} S_{i}\right)$ isomorphically to $A_{n-1}=\bigoplus_{i=1}^{r-1} \pi_{n-1}\left(S_{e}^{i}\right)$, a $\pi$-basis for $\pi_{n-1} M$. If $\pi_{q} M$ has a $\pi$-basis, then an element of $H_{n-1}\left(M ; \pi_{q} M\right) \simeq H_{n-1}\left(\tilde{M} ; A_{q}\right)$ (by Lemma 2.A.2) can be represented as $\sum_{i=1}^{r-1} \sum_{j=1}^{N} a_{i j}\left(g_{i j}\left[S_{e}^{i}\right]\right)=\sum_{i=1}^{r-1}\left(\sum_{j=1}^{N} a_{i j} g_{i j}\right)\left[S_{e}^{i}\right]=$ $\sum_{i=1}^{r-1} x_{i}\left[S_{e}^{i}\right]$, where $x_{i} \in \pi_{q} M$.

3. Calculations of $\pi_{q}(E M)$. All cohomology will be with local coefficients.

3.A. An exact sequence for $\pi_{q}(E M)$.

THEOREM 3.A.1. For $1 \leqslant q \leqslant 2 n-5$ there is an exact sequence

$$
\begin{aligned}
\ldots & \rightarrow H^{1}\left(M ; \pi_{q+2} M\right) \stackrel{D_{q}}{\rightarrow} H^{n}\left(M ; \pi_{n+q} M\right) \rightarrow \pi_{q}(E M) \\
& \rightarrow H^{1}\left(M ; \pi_{q+1} M\right) \stackrel{D_{q-1}}{\rightarrow} H^{n}\left(M ; \pi_{n+q-1} M\right) \rightarrow \ldots
\end{aligned}
$$

Proof. Using the diagram of fibrations discussed in the introduction with $Y=M$, and noting that for $q \geqslant 1, \pi_{q}\left(M^{[M, \varnothing]}\right)=\pi_{q}(E M)$, we obtain an exact sequence for each $q \geqslant 1$ :

$$
J_{q} \stackrel{D_{q}}{\rightarrow} K_{q} \rightarrow \pi_{q}(E M) \rightarrow J_{q-1} \stackrel{D_{q-1}}{\rightarrow} K_{q-1}
$$


In this sequence,

$$
\begin{aligned}
& J_{q}=\operatorname{coker}\left(\pi_{q+1}\left(M^{\left[M^{2}, M^{1}\right]}\right) \rightarrow \pi_{q+1}\left(M^{\left[M^{2}, \varnothing\right]}\right)\right), \\
& K_{q}=\operatorname{coker}\left(\partial: \pi_{q+1}\left(M^{\left[M^{2}, M^{\prime}\right]}\right) \rightarrow \pi_{q}\left(M^{\left[M, M^{2}\right]}\right)\right),
\end{aligned}
$$

and $D_{q}$ is induced by $\partial: \pi_{q+1}\left(M^{\left[M^{2}, \varnothing\right]}\right) \rightarrow \pi_{q}\left(M^{\left[M, M^{2}\right]}\right)$. The theorem is immediate from the following two lemmas.

LEMMA 3.A.2. For $q \geqslant 0, J_{q} \cong H^{1}\left(M ; \pi_{q+2} M\right)$.

Proof. By Lemma 2.B.2, $\pi_{q+1}\left(M^{\left[M^{2}, \star\right]}\right) \rightarrow \pi_{q+1}\left(M^{\left[M^{2}, \phi\right]}\right)$ is surjective. Therefore Lemma 1.B.1 applies.

LEMMA 3.A.3. For $0 \leqslant q \leqslant 2 n-5, K_{q} \cong H^{n}\left(M ; \pi_{n+q} M\right)$.

Proof. By Lemma 2.A.4, $\pi_{p+q} M$ has a $\pi$-basis for $2<p+q<3 n-6$. Therefore when $3<p \leqslant n-1$, the condition $0<q<2 n-5$ guarantees that $\pi_{p+q}(M)$ has a $\pi$-basis. By Lemma 2.A.3, $H^{p}\left(M ; \pi_{p+q} M\right)=H^{p-1}\left(M ; \pi_{p+q} M\right)=0$, so Lemma 1.C.1 applies.

Corollary 3.A.4. For $1 \leqslant q \leqslant n-4, \pi_{q} E M \cong A_{n+q}$, hence is finite.

Proof. For these dimensions, $A_{q+2}=0=A_{q+1}$ by Lemma 2.A.4. Therefore $H^{1}\left(M ; \pi_{q+2} M\right)=0=H^{1}\left(M ; \pi_{q+1} M\right)$, so $\pi_{q}(E M) \simeq H^{n}\left(M ; \pi_{n+q} M\right) \simeq A_{n+q}$, using Lemma 2.A.3.

3.B. Calculation of $D_{q}$. To compute $D_{q}$, we first define a homomorphism $k$ : $H^{1}\left(M ; \pi_{q+2} M\right) \rightarrow \pi_{q+1}\left(M^{\left[M^{2}, \varnothing\right]}\right)$ such that $d_{1} \circ k=$ identity, where $d_{1}$ is the homomorphism of Lemma 1.B.1. Recall the cell structure for $M$ described in $\$ 2$.C. Given a generator $x_{i}\left[S^{i}\right] \in H_{n-1}\left(M ; \pi_{q+2} M\right) \cong H^{1}\left(M ; \pi_{q+2} M\right)$, let $f:\left(\left(M-\operatorname{int}\left(X_{i}\right)\right) \cup\right.$ $\left.\sigma_{i}\right) \times I^{q+1} \rightarrow M$ be a map such that

$$
\left.f\right|_{\left(M-\operatorname{int}\left(X_{i}\right)\right) \times I^{q+1}}=\operatorname{proj}_{M} \text {, and } d_{q+2}\left(\operatorname{proj}_{M}, f\right)\left[\sigma_{i} \times I^{q+1}\right]=x_{i} \in \pi_{q+2}(M, *) .
$$

Define $k\left(x_{i}\left[S^{i}\right]\right)=\left\langle\left. f\right|_{M^{2} \times I^{q+1}}\right\rangle$.

Since $D_{q}$ is induced by $\partial: \pi_{q+1}\left(M^{\left[M^{2}, \varnothing\right]}\right) \rightarrow \pi_{q}\left(M^{\left[M, M^{2}\right]}\right)$, we have $D_{q}=d_{n} \circ \partial \circ k$, where $d_{n}: \pi_{q}\left(M^{\left[M, M^{2}\right]}\right) \rightarrow H^{n}\left(M ; \pi_{n+q} M\right)$ is defined in Lemma 1.C.1. The calculation of $\partial\left\langle\left. f\right|_{M^{2} \times I^{q+1}}\right\rangle$ is exactly analogous to the calculation of $D_{q}$ in the example of $\$ 1 . D$. The generator $z$ there of $\pi_{n-1}(X)$ corresponds to the element $z_{e}^{i}$ of $\pi_{n-1}(M, *)$ (defined in $\left.\$ 2 . A\right)$. The group $\pi_{q+2} X$ is replaced by $\pi_{q+2}(M, *)$ and $\pi_{q}\left(X^{[X, \sigma \cup \partial X]}\right)$ is replaced by $\pi_{q}\left(M^{\left[M, M^{2}\right)}\right)$. Therefore $\partial k\left(x_{i}\left[S^{i}\right]\right)$ is representable by a map $\bar{f}$ which equals $\operatorname{proj}_{M}$ on $\left(M-\left(\right.\right.$ small ball in $\left.\left.X_{i}\right)\right) \times I^{q}$ and such that $d_{n+q}\left(\operatorname{proj}_{M}, \bar{f}\right)\left(\left[M \times I^{q}, M \times \partial I^{q}\right]\right)=\left[z_{e}^{i}, x_{i}\right]$. Hence $D_{q}\left(x_{i}\left[S^{i}\right]\right)=d_{n}\langle\bar{f}\rangle=$ $\left\{\left[z_{e}^{i}, x_{i}\right]\right\}$ where the curly brackets indicate an equivalence class in $H^{n}\left(M ; \pi_{n+q} M\right)$ $\cong \pi_{n+q}(M) / \pi_{1}(M) \cong A_{n+q}$. We have shown

Proposition 3.B.1. $D_{q}\left(\sum_{i=1}^{r-1} x_{i}\left[S^{i}\right]\right)=\sum_{i=1}^{r-1}\left\{\left[z_{e}^{i}, x_{i}\right]\right\} \in A_{n+q}$.

We will now determine $\operatorname{ker} D_{n-3}$ and coker $D_{n-3}$ using the results and notation of $\S 2$.A. For $q=n-3$, we have $\left[z_{e}^{i}, z_{\gamma}^{j}\right]=z_{e, \gamma}^{i, j}$, so 


$$
\begin{aligned}
D_{n-3}: H_{n-1}\left(M ; \pi_{n-1} M\right) \cong & \bigoplus_{i=1}^{r-1} \pi_{n-1} M \rightarrow A_{2 n-3} \\
= & \left(\bigoplus_{i=1}^{r-1} \pi_{2 n-3}\left(S_{e}^{i}\right)\right) \oplus\left(\bigoplus_{i=1}^{r-1} \bigoplus_{\gamma \in \Gamma} \pi_{2 n-3}\left(S_{e, \gamma}^{i, i}\right)\right) \\
& \bigoplus\left(\bigoplus_{1<i<j<r-1} \bigoplus_{\gamma \in \pi_{1} M} \pi_{2 n-3}\left(S_{e, \gamma}^{i, j}\right)\right)
\end{aligned}
$$

given on generators by $D_{n-3}\left(0, \ldots,\left(z_{\gamma}^{j}\right)_{i}, \ldots, 0\right)=\left\{z_{e, \gamma}^{i, j}\right\}$ (where ()$_{i}$ indicates that $z_{\gamma}^{j}$ appears in the $i$ th slot) in all cases except both $\gamma=e$ and $i=j$. We will describe the inverse image of each of these summands in order to determine the kernel and cokernel of $D_{n-3}$. Let $B_{n-3}^{i}=\operatorname{ker}\left(\left.D_{n-3}\right|_{\pi_{n-1}\left(S_{e}^{i}\right)}=\left[z_{e}^{i},-\right]: \pi_{n-1}\left(S_{e}^{i}\right) \rightarrow \pi_{2 n-3}\left(S_{e}^{i}\right)\right)$, which is 0 if $n$ is odd and has index $<2$ if $n$ is even. Let $C_{n-3}^{i}=$ $\operatorname{coker}\left(\left.D_{n-3}\right|_{\pi_{n-1}\left(S_{e}^{i}\right)}\right)$, which is well known to be finite. Observe that $\bigoplus_{l=1}^{r-1} \bigoplus_{\gamma \in \Gamma} \pi_{2 n-3}\left(S_{e, \gamma}^{l, l}\right)$ is in the image of $D_{n-3}$ since $D_{n-3}\left(0, \ldots,\left(z_{\gamma}^{i}\right)_{i}, \ldots, 0\right)=$ $\left\{z_{e, \gamma}^{i, i}\right\}$, and the inverse image of $\left\{z_{e, \gamma}^{i, i}\right\}$ consists of $\left(0, \ldots,\left(z_{\gamma}^{i}\right)_{i}, \ldots, 0\right)$ and $\left(0, \ldots,(-1)^{n-1}\left(z_{\gamma-1}^{i}\right)_{i}, \ldots, 0\right)$. Therefore the kernel contains $\bigoplus_{\gamma \in \Gamma}(Z)_{\gamma}$, where $(\mathbf{Z})_{\gamma} \cong \mathbf{Z}$ is generated by $\left(0, \ldots,\left(z_{\gamma}^{i}-(-1)^{n-1} z_{\gamma-1}^{i}\right)_{i}, \ldots, 0\right)$. Explicitly, we have

$$
\begin{aligned}
D_{n-3}\left(0, \ldots,\left(z_{\gamma}^{i}\right.\right. & \left.\left.-(-1)^{n-1} z_{\gamma-1}^{i}\right)_{i}, \ldots, 0\right)=\left\{\left[z_{e}^{i}, z_{\gamma}^{i}\right]\right\}-\left\{(-1)^{n-1}\left[z_{e}^{i}, z_{\gamma-1}^{i}\right]\right\} \\
& =\left\{\left[z_{e}^{i}, z_{\gamma}^{i}\right]\right\}-\left\{\left[z_{\gamma-1}^{i}, z_{e}^{i}\right]\right\}=\left\{\left[z_{e}^{i}, z_{\gamma}^{i}\right]\right\}-\left\{\gamma^{-1}\left[z_{e}^{i}, z_{\gamma}^{i}\right]\right\}=0 .
\end{aligned}
$$

Finally, $\bigoplus_{\gamma \in \pi} \bigoplus_{1<i<j<r-1} \pi_{2 n-3}\left(S_{e, \gamma}^{i, j}\right)$ is in the image of $D_{n-3}$ and the kernel of the inverse image of $\left\{z_{e, \gamma}^{i, j}\right\}$ is generated by

Explicitly, we have

$$
\left(0, \ldots,\left(z_{\gamma}^{j}\right)_{i}, \ldots,\left(-(-1)^{n-1} z_{\gamma-1}^{i}\right)_{j}, \ldots, 0\right) \text {. }
$$

$$
\begin{aligned}
D_{n-3}\left(0, \ldots, z_{\gamma}^{j}, \ldots,-(-1)^{n-1} z_{\gamma-1}^{i}, \ldots, 0\right) \\
\quad=\left\{\left[z_{e}^{i}, z_{\gamma}^{j}\right]\right\}-\left\{(-1)^{n-1}\left[z_{e}^{j}, z_{\gamma-1}^{i}\right]\right\} \\
\quad=\left\{\left[z_{e}^{i}, z_{\gamma}^{j}\right]\right\}-\left\{\left[z_{\gamma-1}^{i}, z_{e}^{j}\right]\right\}=\left\{\left[z_{e}^{i}, z_{\gamma}^{j}\right]\right\}-\left\{\gamma^{-1}\left[z_{e}^{i}, z_{\gamma}^{j}\right]\right\}=0 .
\end{aligned}
$$

Collecting this information, we state

LEMMA 3.B.2. $\operatorname{coker}\left(D_{n-3}\right) \cong \bigoplus_{i=1}^{r-1} C_{n-3}^{i}$ and

$$
\operatorname{ker}\left(D_{n-3}\right) \cong\left(\bigoplus_{i=1}^{r-1} B_{n-3}^{i}\right) \oplus\left(\bigoplus_{\gamma \in \Gamma} \bigoplus_{i=1}^{r-1} \mathbf{Z}\right) \oplus\left(\bigoplus_{\gamma \in \pi} \underset{1<i<j<r-1}{\bigoplus} \mathbf{Z}\right) .
$$

Corollary 3.B.3. For $n \geqslant 4, \pi_{n-3} E M$ is finite.

Proof. In this case, the exact sequence of Theorem 3.A.1 yields $\pi_{n-3} E M \simeq$ $\operatorname{coker}\left(D_{n-3}\right) \cong \bigoplus_{i=1}^{r-1} C_{n-3}^{i}$.

COROLlARY 3.B.4. $\pi_{n-2} E M$ is infinitely-generated as an abelian group.

Proof. In Theorem 3.A.1 we have a surjection $\pi_{n-2}(E M) \rightarrow \operatorname{ker}\left(D_{n-3}\right)$ and $\operatorname{ker}\left(D_{n-3}\right)$ is infinitely-generated by Lemma 3.B.2.

COROLlary 3.B.5. Let $M^{3}$ be a connected sum of aspherical 3-manifolds. Then $\pi_{1}\left(E M^{3}\right)$ is infinitely-generated. 
Proof. Take $n=3$ in Corollary 3.B.4.

4. Homeomorphisms of nonirreducible 3-manifolds. Let $H X$ denote the path component of $\mathrm{id}_{X}$ in the group $\operatorname{Homeo}(X)$; then $H X \subset E X$. Throughout this section, we will assume $M=M_{1} \# \cdots \# M_{r}$ is a connected sum of $r \geqslant 3$ aspherical 3-manifolds.

From Theorem 3.A.1 we have a homomorphism

$$
D_{0}: H^{1}\left(M ; \pi_{2} M\right) \rightarrow H^{3}\left(M ; \pi_{3} M\right)
$$

and a surjective homomorphism $j: \pi_{1}(E M) \rightarrow \operatorname{kernel}\left(D_{0}\right)$. Let $i: \pi_{1}\left(H M, \operatorname{id}_{M}\right) \rightarrow$ $\pi_{1}\left(E M, \mathrm{id}_{M}\right)$ denote the homomorphism induced by inclusion. The remainder of this section will be devoted to the proof of

THEOREM 4.1. The image of $j \circ i$ contains an infinitely-generated direct summand of kernel $\left(D_{0}\right)$. Hence $\pi_{1}(H M)$ is not finitely-generated.

4.A. Isotopies of $S^{2} \times I$. Let $X=S^{2} \times I$. We regard $S^{2}$ as the unit sphere in $R^{3}$. Since $S O(3)$ preserves $S^{2}$ we have $S O(3) \subset H S^{2}$ (it is actually a deformation retract $[\mathbf{K}])$. Let $S O(2) \subset S O(3)$ be the subgroup of rotations that leave the points $(0,0,1)$ and $(0,0,-1)$ fixed. Let $\tau:(I, 0,1) \rightarrow(S O(2)$, id, id) be the path such that $\tau(t)$ is rotation through an angle of $2 \pi t$; then $\tau$ represents a generator of $\pi_{1}(S O(2))$ $\cong \mathbf{Z}$ (and hence represents a generator of $\pi_{1}(S O(3)) \cong \mathbf{Z} / 2 \mathrm{Z}$ ). We will now use the results and notation of $\S 1$.D. We define a level-preserving homeomorphism $f$ : $X \rightarrow X$ by $f(x, s)=(\tau(s)(x), s)$. Assuming that the 1-cell $\sigma$ equals $(0,0,1) \times I$, we see that $f$ represents an element of $\pi_{0}\left(X^{[X, \partial X \cup \sigma]}\right) \cong \pi_{3}(X) \cong \mathbf{Z}$. It is known that the difference class $d_{3}\left(f, \mathrm{id}_{X}\right)$ is a generator of this group (see [H1, p. 85]). In the exact sequence of $\S 1 . D$,

$$
\pi_{2} X \stackrel{[z,-]}{\rightarrow} \pi_{3} X \rightarrow \pi_{0}\left(X^{[X, \partial X]}\right) \rightarrow 0
$$

the homomorphism $[z,-]$ is well known to have image $2 \mathbf{Z} \subset \mathbf{Z}$; hence $\pi_{0}\left(X^{[X, \partial X]}\right)$ is isomorphic to $\mathbf{Z} / 2 \mathbf{Z}$ and $f$ represents a generator of this quotient group. Now if $\boldsymbol{\theta}$ : $(I \times I, \partial I \times I) \rightarrow(S O(3)$, id $)$ is a nullhomotopy with $\theta(s, 0)=\tau^{2}(s), \theta(s, 1)=\mathrm{id}$, then $F: X \times I \rightarrow X$ defined by $F_{t}(x, s)=F((x, s), t)=(\theta(s, t)(x), s)$ is an isotopy from $f^{2}$ to $\mathrm{id}_{X}$. Under the identification $\pi_{1}\left(X^{[\sigma \cup \partial X, \partial X]}\right) \simeq \pi_{2}(X)$, the restriction $\left.F\right|_{o \cup \partial X}$ represents a generator since $\partial\left\langle\left. F\right|_{\sigma}\right\rangle=\left\langle F_{0}\right\rangle=\left\langle f^{2}\right\rangle$. We choose the generator $z$ of $\pi_{2}(X)$ such that $d_{1}\left(\left\langle\left. F\right|_{\sigma}\right\rangle\right)([\sigma \times I])=z$. Note that $F^{-1}$ is an isotopy from $\left(f^{-1}\right)^{2}$ to id ${ }_{X}$, with $d_{1}\left(\left\langle\left. F^{-1}\right|_{\sigma}\right\rangle\right)([\sigma \times I])=-z$.

4.B. The 3-manifold $Z$. Let $X_{1}$ and $X_{2}$ be two copies of $S^{2} \times I$, and let $B=D^{2} \times I$. Let $Z$ denote the 3-manifold-with-boundary obtained by identifying $D_{1}=D^{2} \times\{0\}$ with a disc in $S^{2} \times\{1\} \subset \partial X_{1}$ and $D_{2}=D^{2} \times\{1\}$ with a disc in $S^{2} \times\{1\} \subset \partial X_{2}$, by orientation-reversing homeomorphisms. We assume these discs do not contain the 0-cells $\sigma_{1} \cap S^{2} \times\{1\} \subset \partial X_{1}$ and $\sigma_{2} \cap S^{2} \times\{1\} \subset \partial X_{2}$. We will use $S_{1}$ to denote $S^{2} \times\{0\} \subset \partial X_{1}$ and $S_{2}$ for $S^{2} \times\{0\} \subset \partial X_{2}$. Let $S_{3}$ denote the remaining boundary component of $Z$, so that the oriented boundary of $Z$ is $\partial Z=S_{1} \cup S_{2} \cup\left(-S_{3}\right)$. We choose a nice collar neighborhood $X_{3}$ of $S_{3}$ so that $\sigma_{1} \cap X_{3}=\sigma_{1}$ has the form $\left(\sigma_{1} \cap S_{3}\right) \times I=(0,0,1) \times I$ and $\sigma_{2} \cap X_{3}=\sigma_{2}$ 
has the form $\left(\sigma_{2} \cap S_{3}\right) \times I=(0,0,-1) \times I$. We will define two isotopies of $Z$. The isotopy $G$ is defined by

$$
G_{t}(z)=G(z, t)= \begin{cases}F_{t}(x, s) & \text { if } z=(x, s) \in X_{3}, \\ z & \text { if } z \notin X_{3},\end{cases}
$$

and the isotopy $H$ is defined by

$$
H_{t}(z)=H(z, t)= \begin{cases}F_{t}(x, s) & \text { if } z=(x, s) \in X_{1}, \\ F_{t}^{-1}(x, s) & \text { if } z=(x, s) \in X_{2}, \\ z & \text { if }(z, t) \in B \times I .\end{cases}
$$

We make the important observation that $G_{0}$ is isotopic to $H_{0}$ by an isotopy which is fixed on $\sigma_{1} \cup \sigma_{2} \cup \partial Z$. This observation appears in the thesis of Hendriks [H1, p. 103].

We will now construct a nontrivial element of $\pi_{1}\left(Z^{[Z, \partial Z)}\right)$. Let $\sigma=\sigma_{1} \cup \sigma_{2} \subset Z$. From the fibration $Z^{[Z, \sigma \cup \partial Z]} \rightarrow Z^{[Z, \partial Z]} \rightarrow Z^{[\sigma \cup \partial Z, \partial Z]}$ we obtain a commutative diagram

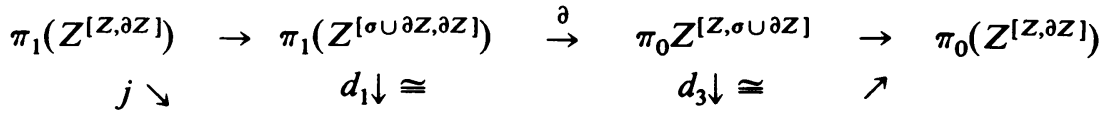

$$
\begin{aligned}
& H^{1}\left(Z, \partial Z ; \pi_{2} Z\right) \stackrel{D_{0}}{\rightarrow} H^{3}\left(Z, \partial Z ; \pi_{3} Z\right)
\end{aligned}
$$

Now $Z \simeq S_{1} \vee S_{2}$, so using the results of Hilton [H2] we may write $\pi_{2}(Z) \cong \pi_{2}\left(S_{1}\right)$ $\bigoplus \pi_{2}\left(S_{2}\right)$ and $\pi_{3}(Z) \cong \pi_{3}\left(S_{1}\right) \oplus \pi_{3}\left(S_{2}\right) \oplus \pi_{3}\left(S_{1,2}\right)$ where $S_{1,2}$ is a 3-sphere. Let $z_{1}, z_{2}$ be generators of $\pi_{2}\left(S_{1}\right)$ and $\pi_{2}\left(S_{2}\right)$, respectively, such that the homotopy class represented by the oriented sphere $S_{3}$ equals $z_{1}+z_{2} \in \pi_{2}(Z)$. Then the Whitehead product $z_{1,2}=\left[z_{1}, z_{2}\right]$ corresponds to a generator of $\pi_{3}\left(S_{1,2}\right) \subset \pi_{3}(Z)$. Let $z_{i, i}, 1 \leqslant i \leqslant 2$, be the generators of $\pi_{3}\left(S_{i}\right)$ so that $\left[z_{i}, z_{i}\right]=2 z_{i, i}$. We have $H^{1}\left(Z, \partial Z ; \pi_{2} Z\right) \cong \mathbf{Z} \oplus \mathbf{Z} \oplus \mathbf{Z} \oplus \mathbf{Z}$ where the summands are generated by the cocycles $d_{i, j}, 1 \leqslant i, j \leqslant 2$, such that $d_{i, j}\left[\sigma_{i} \times I\right]=z_{j}$ and $d_{i, j}\left[\sigma_{k} \times I\right]=0$ if $k \neq i$. We also have $H^{3}\left(Z, \partial Z ; \pi_{3} Z\right) \cong \pi_{3}(Z) \cong \mathbf{Z} \oplus \mathbf{Z} \oplus \mathbf{Z}$ generated by the cocycles $c_{1,1}, c_{2,2}$, and $c_{1,2}$, where $c_{1,1}([Z, \partial Z])=z_{1,1}$ and so on. Let $c_{2,1}=c_{1,2}$. As in $\$ 3 . \mathrm{B}$, it follows that the homomorphism $D_{0}$ is given by $D_{0}\left(d_{i, j}\right)=c_{i, j}$ if $i \neq j$ while $D_{0}\left(d_{i, i}\right)$ $=2 c_{i, i}$. Therefore the kernel of $D_{0}$ is generated by $d_{1,2}-d_{2,1}$. From the discussion of $F: X \times I \rightarrow X$, we have $d_{1}\left\langle\left. H\right|_{\sigma}\right\rangle=d_{1,1}-d_{2,2}$. To find $d_{1}\left\langle\left. G\right|_{\sigma}\right\rangle$, we see from the definition of $G$ that $d_{1}\left\langle\left. G\right|_{\sigma}\right\rangle\left(\left[\sigma_{1} \times I\right]\right)=z_{1}+z_{2}$; hence $d_{1}\left\langle\left. G\right|_{\sigma}\right\rangle=d_{1,1}+d_{1,2}$ $+\alpha d_{2,1}+\beta d_{2,2}$ for some $\alpha, \beta \in Z$. Since $G_{0}$ is isotopic to $H_{0}$ with $\sigma$ held fixed, we have $D_{0}\left(d_{1}\left(\left\langle\left. G\right|_{\sigma}\right\rangle\right)\right)=D_{0}\left(d_{1}\left(\left\langle\left. H\right|_{\sigma}\right\rangle\right)\right)=2 c_{1,1}-2 c_{2,2}$. From our formula for $D_{0}$ we also have

$$
\begin{aligned}
D_{0}\left(d_{1}\left(\left\langle\left. G\right|_{\sigma}\right\rangle\right)\right) & =D_{0}\left(d_{1,1}+d_{1,2}+\alpha d_{2,1}+\beta d_{2,2}\right) \\
& =2 c_{1,1}+c_{1,2}+\alpha c_{2,1}+2 \beta c_{2,2} ;
\end{aligned}
$$

hence $\alpha=\beta=-1$ and $d_{1}\left(\left\langle\left. G\right|_{\sigma}\right\rangle\right)\left(\left[\sigma_{2} \times I\right]\right)=-z_{1}-z_{2}$. The identity map of $Z$ is isotopic to $G_{0} \circ H_{0}^{-1}$ by an isotopy which is fixed on $\sigma \cup \partial Z$. Define an isotopy $J$ : $Z \times I \rightarrow Z$ so that for $0 \leqslant t<\frac{1}{2}, J$ is such an isotopy, while $J(z, t)=$ $\left(G_{2 t-1} \circ H_{2 t-1}^{-1}\right)(z, t)$ for $\frac{1}{2} \leqslant t \leqslant 1$. Then $J$ is a loop in $\operatorname{Homeo}(Z)$ and regarding $J$ 
as a loop in $Z^{[Z, \partial Z]}$ we have $d_{1}\left\langle\left. J\right|_{0}\right\rangle=d_{1}\left\langle\left. G\right|_{\sigma}\right\rangle-d_{1}\left\langle\left. H\right|_{0}\right\rangle=d_{1,2}-d_{2,1}$. That is, $j\langle J\rangle$ is a generator of $\operatorname{kernel}\left(D_{0}\right)^{1}$

4.C. Isotopies of $M$. To construct isotopies of $M$, we will use the cell-complex structure of $M$ defined in \$2.C. Since $\bigvee_{i=1}^{r-1} X_{i} \subset M$ is simply-connected and contains the basepoint $*$ of $M$, a path in $M$ with endpoints in $\bigvee_{i=1}^{r-1} X_{i}$ represents a well-defined element of $\pi_{1}(M, *)$. We may represent any element $\langle\alpha\rangle \in$ $\pi_{1}\left(M_{3}\right) * \cdots * \pi_{1}\left(M_{r}\right) \subset \pi_{1}(M)$ by a nicely-imbedded $\operatorname{arc} \alpha$ in $M$ that runs from $X_{1}$ to $X_{2}$ intersecting them only in its boundary. We can imbed $Z$ in $M$ so that

1. $X_{1} \subset Z$ is mapped homeomorphically to $X_{1} \subset M$, carrying the basepoint $\sigma_{1} \cap S_{3}$ to *.

2. $D^{2} \times I \subset Z$ is mapped to a tubular neighborhood of $\alpha$ that intersects $X_{1} \cup X_{2}$ in $D^{2} \times \partial I$.

3. $X_{2} \subset Z$ is mapped homeomorphically into $X_{2}-*$. (This will reverse the local orientation, when $\alpha$ is orientation-reversing.)

Such an imbedding induces an injection $\pi_{2}\left(Z, \sigma_{1} \cap S_{3}\right) \rightarrow \pi_{2}(M, *)$ given on generators by $z_{1} \rightarrow z_{e}^{1}$ and $z_{2} \rightarrow z_{\alpha}^{2}$.

The isotopy $J_{\alpha}$ of $M$ is of course defined to be $J$ on $Z \subset M$ and the identity outside Z. Now $d_{1}\left(\left\langle\left. J_{\alpha}\right|_{M^{2}}\right\rangle\right)=d_{\alpha}$ is the element of $H^{1}\left(M ; \pi_{2} M\right)$ such that $d_{\alpha}\left(\left[\sigma_{k} \times I\right]\right)=0$ if $3 \leqslant k \leqslant r-1, d_{\alpha}\left(\left[\sigma_{1} \times I\right]\right)=z_{\alpha}^{2}$, and $d_{\alpha}\left(\left[\sigma_{2} \times I\right]\right)=-z_{\alpha^{-1}}^{1}$. The last formula differs by the action of $\alpha^{-1}$ from the corresponding calculation for $\left\langle\left. J\right|_{\sigma}\right\rangle \in \pi_{1}\left(Z^{[\sigma, \partial \sigma]}\right)$, since in $M$ we use a path in $X_{2}$ to base the homotopy class that is the value of the difference cohomology class $d_{1}\left(J_{\alpha} \operatorname{proj}_{M}\right)$ on $\left[\sigma_{2} \times I\right]$, rather than a path in $Z$ that follows along $\alpha$ back to $X_{1}$. Under the isomorphism $H^{1}\left(M ; \pi_{2} M\right) \cong \bigoplus{ }_{i=1}^{r-1} \pi_{2}(M), d_{1}\left(\left\langle\left. J_{\alpha}\right|_{M^{2}}\right\rangle\right)$ corresponds to $\left(z_{\alpha}^{2},-z_{\alpha^{-1}}^{1}, 0, \ldots, 0\right)$. Regarding the $J_{\alpha}$ as loops in $E M$, we have $j\left\langle J_{\alpha}\right\rangle=\left(z_{\alpha}^{2},-z_{\alpha^{-1}}^{1}, 0, \ldots, 0\right)$; hence the elements $j\left\langle J_{\alpha}\right\rangle, \alpha \in \pi_{3}(M) * \cdots * \pi_{r}(M)$, generate an infinitely-generated summand of kernel $\left(D_{0}\right)$, using Lemma 3.B.2. This concludes the proof of Theorem 4.1.

Question. Can the generators of $\operatorname{kernel}\left(D_{0}\right)$ of the form $\left(z_{\gamma}^{1}-z_{\gamma^{-1}}^{1}, 0, \ldots, 0\right)$, or of the form $\left(z_{\gamma}^{2},-z_{\gamma^{-1}}^{1}, 0, \ldots, 0\right)$ where $\gamma$ involves elements of $\pi_{1} M_{1} * \pi_{1} M_{2}$, be realized as the images $j\left\langle J_{\gamma}\right\rangle$ of loops $J_{\gamma}$ in $H M$ ?

\section{BIBLIOGRAPHY}

[B] E. Bloomberg, Manifolds with no periodic homeomorphisms, Trans. Amer. Math. Soc. 202 (1975), 67-78.

[F] H. Federer, A study of function spaces by spectral sequences, Trans. Amer. Math. Soc. 82 (1956), 340-361.

[F-K] D. Frank and D. W. Kahn, Finite complexes with infinitely-generated groups of self-equivalences, Topology 16 (1977), 189-192.

[G] D. H. Gottlieb, A certain subgroup of the fundamental group, Amer. J. Math. 87 (1965), 840-856.

[H1] H. Hendriks, Sur les équivalences d'homotopie en dimension 3, Dissertation, University of Paris at Orsay, 1975.

[H2] P. J. Hilton, On the homotopy groups of the union of spheres, J. London Math. Soc. 30 (1955), 154-172.

[K] H. Kneser, Die Deformationssätze der einfach zusammenhängenden Flächen, Math. Z. 25 (1926), 362-372.

\footnotetext{
'I learned from Frank Quinn that he has encountered and used the isotopy $J$ in another context.
} 
[M1] D. McCullough, Connected sums of aspherical manifolds, Indiana Univ. Math. J. 29 (1980).

[M2] _ Homotopy groups of the space of self-homotopy equivalences, Dissertation, University of Michigan, 1978.

[M3] __ Finite aspherical complexes with infinitely-generated groups of self-homotopy-equivalences, Proc. Amer. Math. Soc. 80 (1980), 337-340.

[S] D. Sullivan, Infinitesimal computations in topology, Inst. Hautes Étude Sci. Publ. Math. 47 (1977), 269-331.

[W] C. W. Wilkerson, Applications of minimal simplicial groups, Topology 15 (1976), 111-130.

Department of Mathematics, University of OKLahoma, Norman, OKLahoma 73019 